\title{
Plasmon phenomena and luminescence amplification in nanocomposite structures
}

\author{
H. E. Ruda and A. Shik \\ Centre for Advanced Nanotechnology, University of Toronto, Toronto M5S 3E4, Canada \\ (Received 29 November 2004; revised manuscript received 1 February 2005; published 27 June 2005)
}

\begin{abstract}
The optical characteristics (absorption and reflection coefficients) of metal-dielectric mixtures, as well as of nanoshells with such mixture as a shell layer, are considered within the framework of the effective medium approximation. The evolution of plasmon characteristics and optical properties with changes of metal composition in a shell layer and its thickness is studied. The results are applied to the problem of enhancing the luminescence characteristics of semiconductor nanocrystals covered by metallic nanoshells.
\end{abstract}

DOI: 10.1103/PhysRevB.71.245328

PACS number(s): 73.22.Lp, 78.67.Bf, 73.21.La

\section{INTRODUCTION}

Optical properties of nanostructures and nanocomposites are now the object of intensive studies, with the primary attention being paid to interband absorption and luminescence in semiconductor nanocrystals strongly modified by size quantization phenomena. In this work we discuss another, much less investigated group of problems devoted to optical properties of metallic, rather than semiconducting, nanostructures caused by plasmon oscillation in them. We consider plasmon characteristics of metal-dielectric (or metal-semiconductor) nanocomposites and of metallic nanoshells. In the case of nanoshells we trace the evolution of the optical spectra in the process of nanoshell growth beginning from separate disconnected metallic particles towards uniform spherical layers completely covering semiconductor or dielectric core nanocrystals. Special attention is paid to the distribution of light intensity in such core-shell structures and their application for enhancing the photoluminescence efficiency in core semiconductor nanocrystals.

\section{PLASMONS IN METAL-DIELECTRIC MIXTURES}

In the present section we discuss briefly the main properties of plasmons determining optical absorption in metaldielectric mixtures. Some of these results are already known and we give only the survey of those which will be used to derive and explain the formulas of subsequent sections.

It is well known that a single spherical metallic dot exhibits a strong plasmon light absorption at $\omega \simeq \omega_{p} / \sqrt{3}$ where $\omega_{p}$ is the plasma frequency for a bulk metal. Formally this means that the imaginary part of dielectric constant $\varepsilon(\omega)$ has a peak near $\omega_{p} / \sqrt{3}$ with the height and width determined by the relaxation time of electrons in metal. The exact value of the dot radius is irrelevant here, provided it is much less than the wavelength of light having the frequency $\omega$. This kind of a plasmon peak is characteristic also for metal-dielectric mixtures at a very low volume concentration of metal $C$.

With an increase of $C$, mutual influence of metallic particles becomes important. The corresponding modification of plasmon phenomena was investigated in a number of experimental and theoretical works. One very interesting experimental finding was a redshift in the plasmon resonance with increasing concentration or size of metallic islands (see, e.g., Refs. 1-5). Theoretically, the tendency of a decrease in the effective plasmon frequency on approaching two metallic particles was demonstrated using numerical simulation ${ }^{6}$ and within the framework of the Maxwell-Garnett perturbation approach. ${ }^{3,5}$ We consider below optical properties of random metal-dielectric mixtures using the effective medium approximation (EMA), which avoids the restriction of only considering low $C$ but also consider (at least qualitatively) the situation near the percolation threshold when neighboring particles touch and the system as a whole acquires metallic conductivity.

Let us describe the metallic phase by the standard freeelectron expression for dielectric constant $\varepsilon_{M}=1-\left[\omega_{p}^{2} / \omega(\omega\right.$ $-i \nu)]$, where $\nu$ is the scattering rate, and characterize the nonconducting phase by its real dielectric constant $\varepsilon_{D}$. Then, according to the three-dimensional EMA (see, e.g., Ref. 7), the effective $\varepsilon$ of the mixture is given by the expression

$$
\varepsilon_{\mathrm{eff}}^{(3)}=\frac{1}{4}\left(\gamma \pm \sqrt{\gamma^{2}+8 \varepsilon_{M} \varepsilon_{D}}\right), \quad \gamma=(2-3 C) \varepsilon_{D}+(3 C-1) \varepsilon_{M},
$$

where the sign before the radical should be chosen from the requirement that $\operatorname{Im} \varepsilon<0$. If metallic particles are distributed randomly not in the volume but on a plane, it is more suitable to describe their properties using the two-dimensional EMA, ${ }^{8}$ which, contrary to Eq. (1), gives

$$
\begin{aligned}
\varepsilon_{\mathrm{eff}}^{(2)}= & \frac{1}{2}\left[(1-2 C)\left(\varepsilon_{D}-\varepsilon_{M}\right)\right. \\
& \left. \pm \sqrt{(1-2 C)^{2}\left(\varepsilon_{D}-\varepsilon_{M}\right)^{2}+4 \varepsilon_{M} \varepsilon_{D}}\right] .
\end{aligned}
$$

The range of applicability of the EMA formulas (1) and (2) requires a special discussion. It is known that for a very large difference between $\varepsilon_{M}$ and $\varepsilon_{D}$ (e.g., in an ideal metaldielectric mixture) the EMA becomes inadequate in the vicinity of the percolation threshold $C_{p}=1 / 2$ (in two dimensions) or $C_{p}=1 / 3$ (in three dimensions) [note that the twodimensional value $C_{p}=1 / 2$ is an exact one, while the threedimensional $C_{p}=1 / 3$ given by EMA is approximate and exceeds real $C_{p}$ obtained by numerical calculations (see, e.g., Ref. 9)] but if this difference is moderate (in Ref. 7 the estimate $0.05<\left|\varepsilon_{M} / \varepsilon_{D}\right|<20$ is given), the EMA can be ap- 

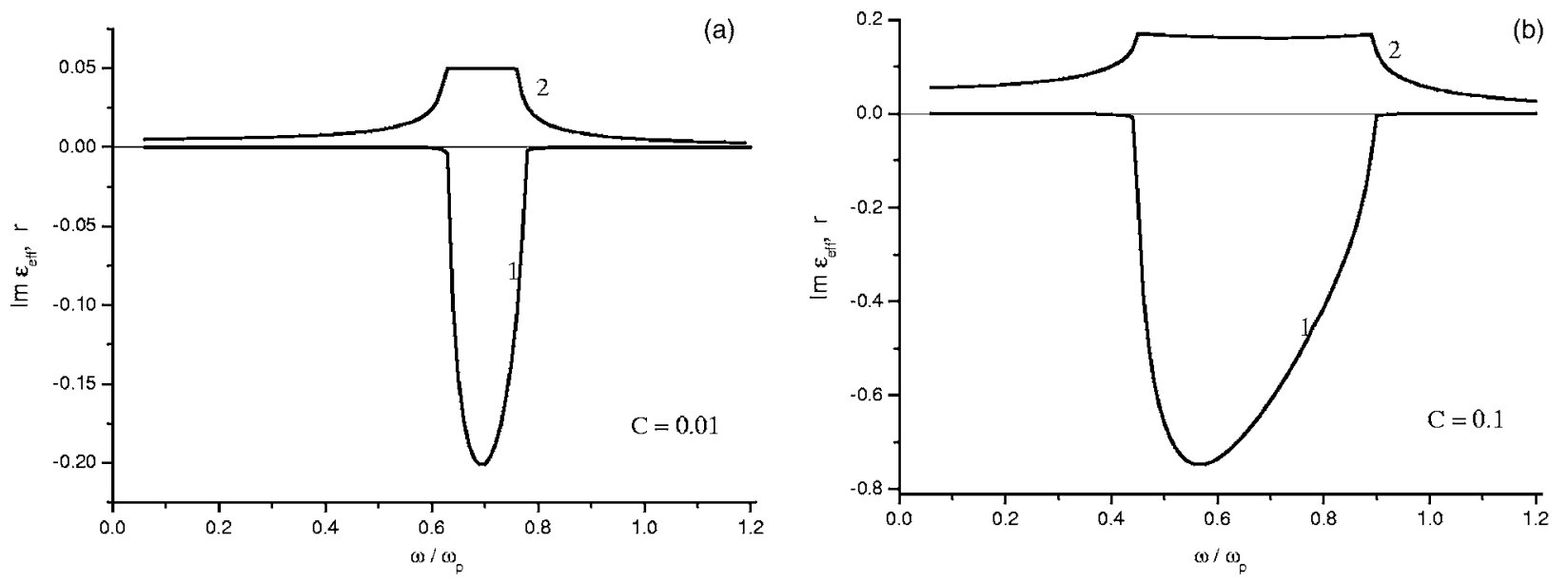

(c)
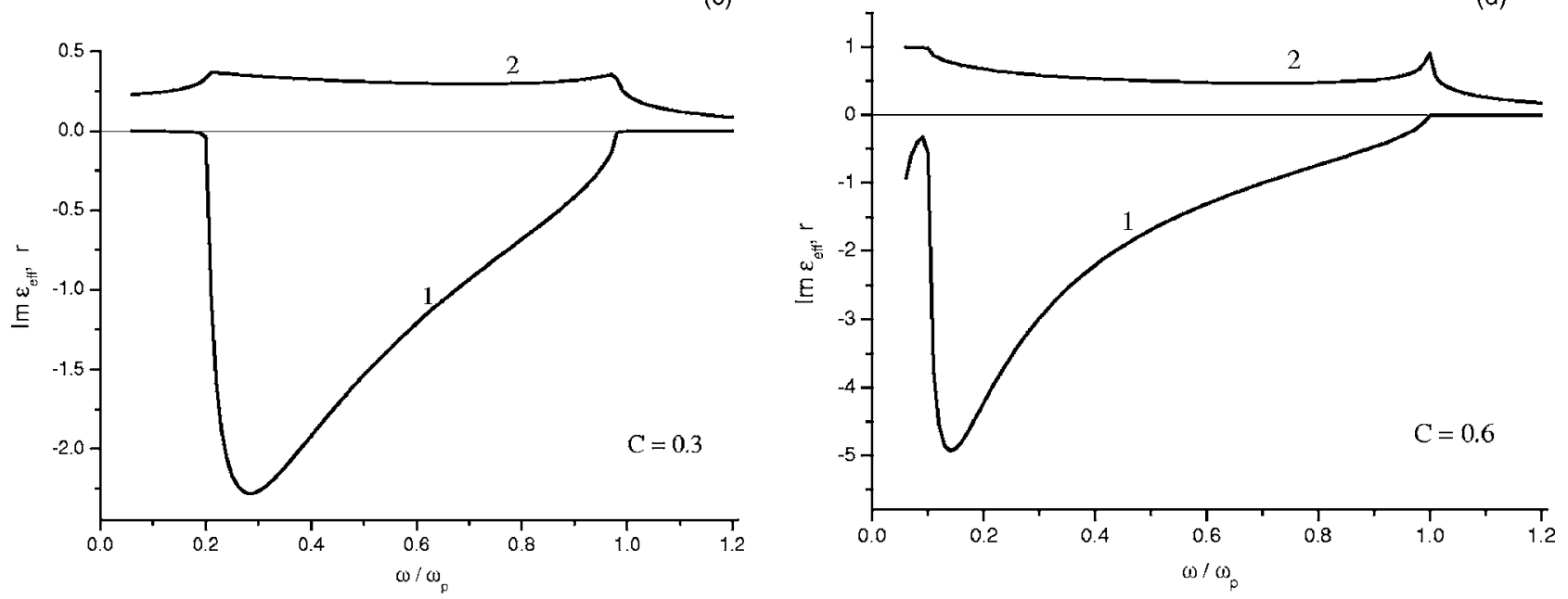

(e)
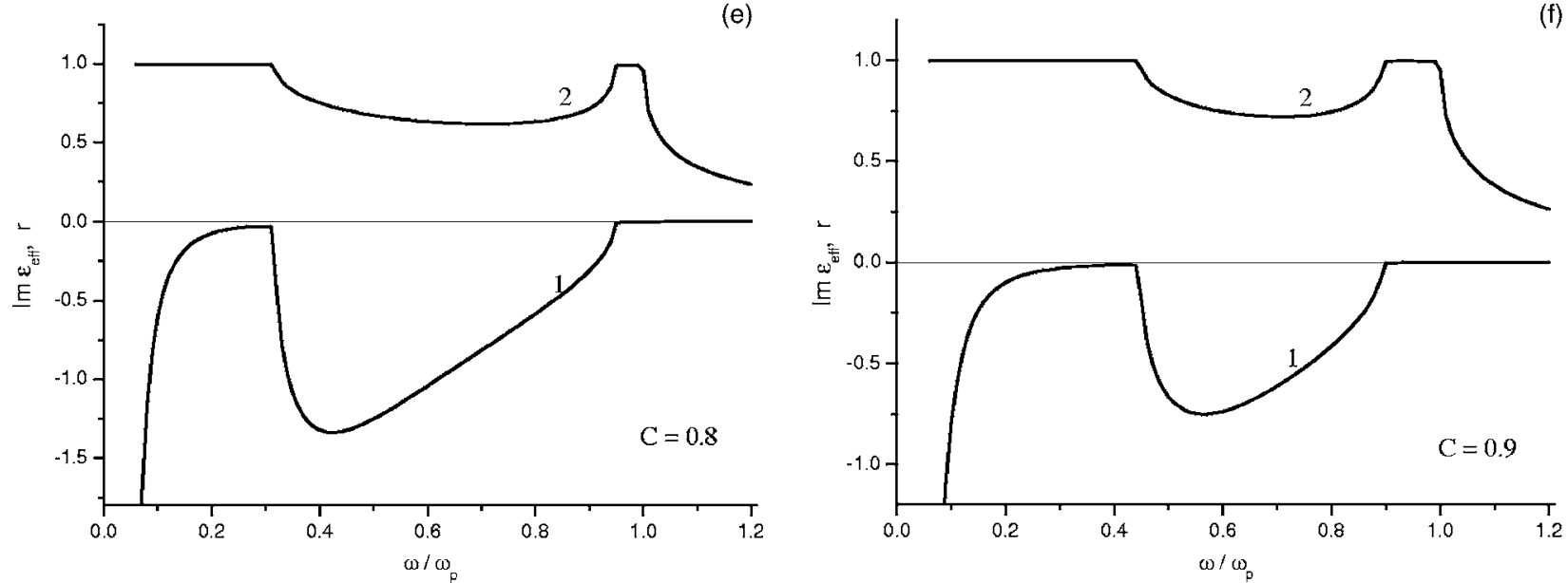

FIG. 1. Spectral dependences of $\operatorname{Im} \varepsilon_{\text {eff }}^{(2)}$ (curves 1) and the reflection coefficient $r$ (curves 2) for metal-dielectric mixtures with $\varepsilon_{D}=1$ at $C=0.01$ (a); 0.1 (b); 0.3 (c); 0.6 (d); 0.8 (e); 0.9 (f).

plied for all possible $C$. For our choice of $\varepsilon_{M}$ and $\varepsilon_{D}$ it can be violated in two spectral regions: $\omega \simeq \omega_{p}$ and $\omega \rightarrow 0$ where $\varepsilon_{M}$ of an ideal metal tends, respectively, to 0 and $\infty$. However, we consider real metallic structures with noticeable carrier scattering assuming $\nu / \omega_{p} \simeq 0.1$ in most of the analysis that follows. In this case, in the most interesting region corresponding to plasmon absorption, applicability of the EMA is never undermined. As to the low frequency limit, it is of no practical interest for our problem, and we restricted our calculations to the region $\omega / \omega_{p}>0.05$.

Figure 1 shows the spectral dependences of $\operatorname{Im} \varepsilon_{\text {eff }}^{(2)}$ (characterizing the absorption coefficient) and the reflection coefficient $r=\left|\left(\sqrt{\varepsilon_{\mathrm{eff}}^{(2)}}-1\right) /\left(\sqrt{\varepsilon_{\mathrm{eff}}^{(2)}}+1\right)\right|$ in a metal-dielectric mixture obtained from Eq. (2) at different $C$. It is seen that the maxi- 


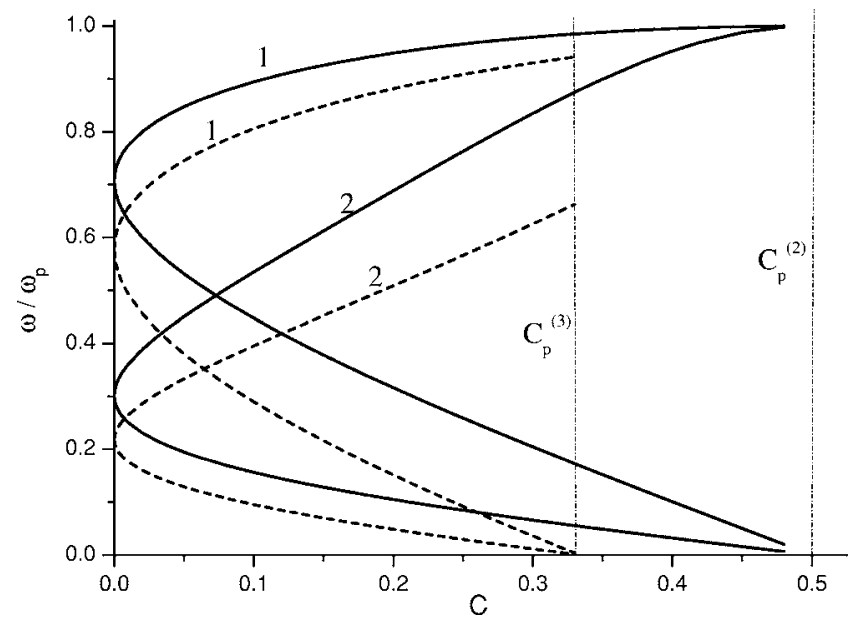

FIG. 2. Regions of plasmon absorption in two- (solid) and threedimensional (dashed) EMA for $\varepsilon_{D}=1$ (curves 1) and $\varepsilon_{D}=10$ (curves $2)$. Vertical lines show the corresponding percolation thresholds.

mum in $|\operatorname{Im} \varepsilon|$ corresponds to the plasmon absorption and is equal to the energy of two-dimensional plasmon $\omega_{p} / \sqrt{2}$ at low $C$; with increasing $C$, the maximum shifts to lower frequencies, in accordance with the abovementioned results. Figures 1(a)-1(c) show that with increasing $C$, the absorption band not only shifts but broadens and becomes asymmetric, also in complete agreement with experiment. ${ }^{5}$ It is worth noting that the absorption is not related to scattering (as in a single metallic sphere), and at small $\nu$ is $\nu$ independent and occurs in the frequency region where the argument of square root in Eqs. (1) and (2) is negative. The boundaries of these regions and their evolution with $C$ are shown in Fig. 2 for two different $\varepsilon_{D}: \varepsilon_{D} \simeq 1$ corresponding to metal particles in air or other low-refractive medium, and $\varepsilon_{D} \simeq 10$ typical for most semiconductors.

When $C$ exceeds the percolation threshold $C_{p}=1 / 2$ corresponding to the onset of static metallic conductivity [Figs. $1(\mathrm{~d})$ and $1(\mathrm{f})$ ], most properties of the mixture become qualitatively similar to those of a bulk metal. That is, they are characterized by a fast growth of $|\varepsilon|$ at small $\omega$ (complete screening of the electric field) and the presence of the plasma reflection spectral regions with $r=1$. The width of these regions increases with $C$ from zero at the percolation level, and at $C \rightarrow 1$ they merge corresponding to the pure metallic situation $\omega<\omega_{p}$. Similar calculations of $\varepsilon_{\mathrm{eff}}^{(3)}$ using Eq. (1) give qualitatively similar results and are not shown in Fig. 1 (though are reflected in Fig. 2). The only quantitative distinctions consist in a different small- $C$ plasmon frequency, equal to $\omega_{p} / \sqrt{3}$ (the well-known result for a single metallic sphere) and a different percolation threshold $C_{p}=1 / 3$.

The model discussed describes the properties not only of metal-dielectric mixtures but also of such interesting object as low-temperature grown GaAs containing metallic As clusters in semiconducting matrix. ${ }^{10}$ Optical absorption spectra in this material were discussed in Ref. 11 for small cluster concentration $C$ while the present work shows their evolution at arbitrary $C$. Note that bulk As has the carrier concentration two orders of magnitude less than typical metals so that for the low-temperature grown GaAs $\omega_{p} \simeq 10^{15} \mathrm{~s}^{-1}$ corresponds to the infrared spectral region.

\section{METALLIC NANOSHELLS}

Metallic nanoshells (a term introduced in Ref. 12) represent one of the most interesting types of metal-dielectric (or metal-semiconductor) nanostructures consisting of a dielectric (or semiconductor) quantum dot covered by a thin metallic shell. The technological feasibility of fabricating nanoshells with nanometer dimensions have been already confirmed by a number of successful experiments (see, e.g., Refs. 12-14). The interest in such structures is stimulated by the fact that optical excitation with the frequency close to the plasmon resonance may generate in the vicinity of a nanostructure, particularly in its core, anomalously high intensity of ac electric field. This phenomenon, having much in common with the surface-enhanced Raman spectroscopy (SERS), ${ }^{15}$ results also in an increase in the optical nonlinearity and photoluminescence of quantum dots, which is especially important for their applications in light-emitting structures ${ }^{16}$ and biolabeling. ${ }^{17}$ The absorption spectra and electric field distribution in such core-shell structures have already been calculated by different authors. ${ }^{18-20}$

However, in real nanoshell structures the metallic shell, especially at first stages of formation, is nonuniform consisting of separate disconnected or partially connected metallic dots. ${ }^{12}$ This changes dramatically the distribution of electric field in the system and its dielectric constant, and will be the subject of analysis in this section.

We consider a spherical quantum dot with radius $r_{1}$ and dielectric constant $\varepsilon_{1}$ covered by a shell with the internal radius $r_{1}$ and the external radius $r_{2}$. The shell represents a metal-dielectric mixture considered in the previous section with air as a dielectric $\left(\varepsilon_{D}=1\right)$. The ambient medium at $r$ $>r_{2}$ also has $\varepsilon=1$. Since the growth of nanoshells starts as a formation of separate metallic islands on a core surface followed by their coalescence, it is more natural to consider the shell as a two-dimensional mixture (see also corresponding discussion in the last section), as described by Eq. (2). By using this formula and the expression for nanoshell polarizability $^{18}$

$$
\alpha=4 \pi r_{2}^{3} \frac{2\left[\varepsilon_{\mathrm{eff}}^{(2)}\right]^{2} P+\varepsilon_{\mathrm{eff}}^{(2)}\left(3 \varepsilon_{1}-2 \varepsilon_{1} P-3+P\right)-\varepsilon_{1} P}{2\left[\varepsilon_{\mathrm{eff}}^{(2)}\right]^{2} P+\varepsilon_{\mathrm{eff}}^{(2)}\left(3 \varepsilon_{1}-2 \varepsilon_{1} P+6-2 P\right)+2 \varepsilon_{1} P}
$$

$\left[P=1-\left(r_{1} / r_{2}\right)^{3}\right.$ is the ratio of shell volume to total particle volume], we can determine the spectral characteristics of the nanoshells under consideration.

The results of the calculations presented in Fig. 3 warrant some additional comments. In fact, applicability of Eq. (2) to nanoshells is not obvious. Such a two-dimensional approach can be adequate either in bulk samples with cylindrical inhomogeneities or in thin layer inhomogeneous systems having electric field confined in the plane of the system, as for transport phenomena under conditions of quasineutrality, considered in the first work of Adkins. ${ }^{8}$ Nanoshells, generally speaking, satisfy neither of these conditions. However, we are interested in the spectral region corresponding to plasmon resonances when the effective dielectric constant of the metallic shell tends to zero. Under these conditions, we may expect the high-frequency electric field to be concen- 

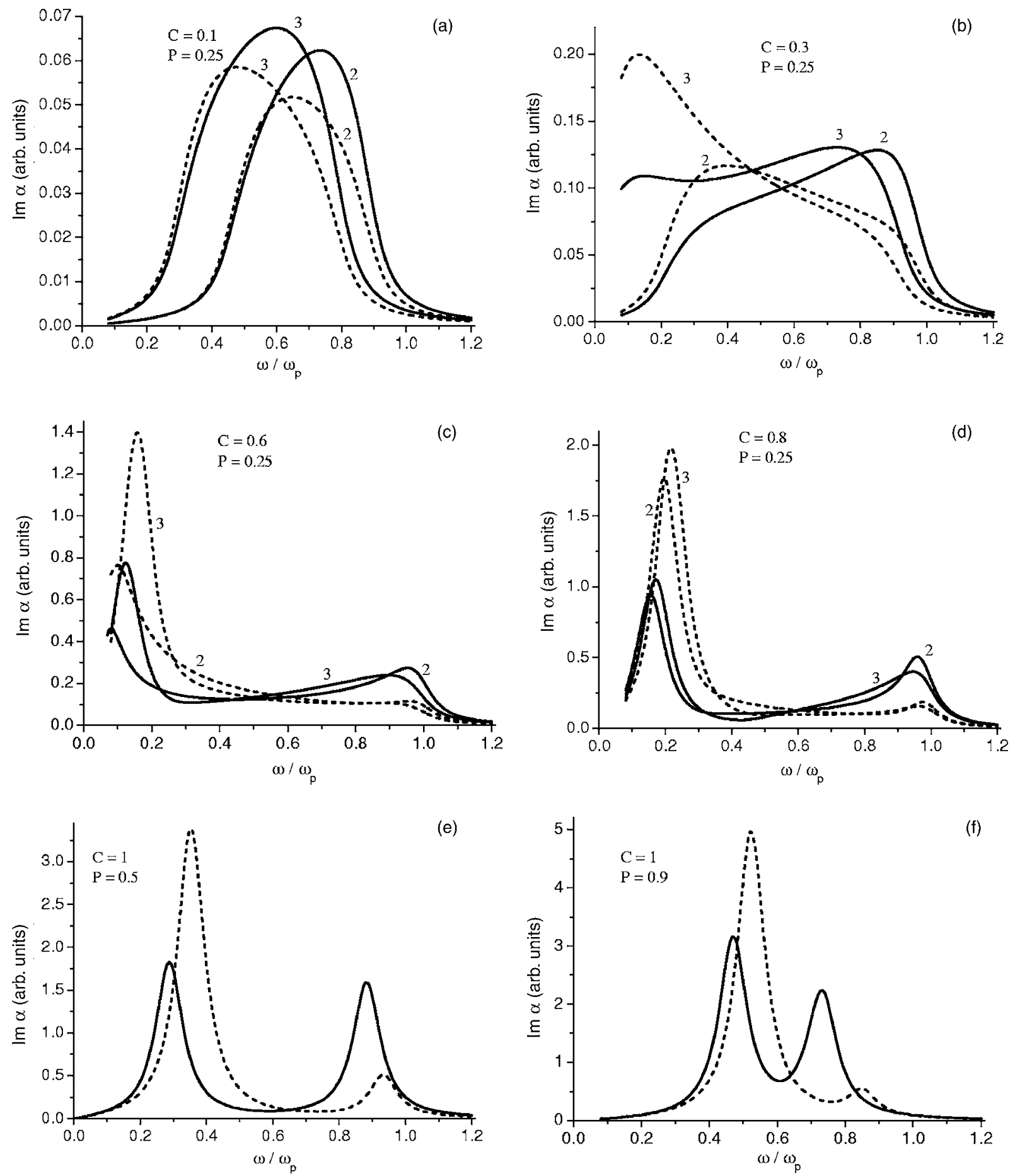

FIG. 3. Absorption spectra of disconnected (a),(b), connected (c),(d), and uniform (e),(f) metallic nanoshells with $C=0.1, P=0.25$ (a); $C=0.3, P=0.25$ (b); $C=0.6, P=0.25$ (c); $C=0.8, P=0.25$ (d); $C=1, P=0.5$ (e); $C=1, P=0.9$ (f). The spectra are given in relative units the same for all figures. Solid curves correspond to $\varepsilon_{1}=3$, dashed curves to $\varepsilon_{1}=1$ (hollow nanoshells). The numerals 2 and 3 beside the curves, correspond to calculations within the two- and three-dimensional EMA, respectively. In cases (e) and (f), these results are completely coincident.

trated mostly in the shell, making our approach correct, at least semiquantitatively. Nevertheless, we performed similar calculations using $\varepsilon_{\text {eff }}^{(3)}$ as well. Comparison of the corresponding curves in Fig. 3 shows that, in spite of quantitative differences at small $C$ and $P$, the qualitative character of their evolution remains the same and is formulated below.
Solid lines (a)-(d) in Fig. 3 show the evolution of the spectral dependence of $\operatorname{Im} \alpha$ (the absorption coefficient) with the increase of metallic content calculated for a shell with the external radius $r_{2}=1.1 r_{1}(P \simeq 0.25)$ and $\varepsilon_{1}=3$. The initial peak around $\omega_{p} / \sqrt{2}$, similar to that in Fig. 1, with the increase in $C$ broadens into an asymmetric wide band with the 
maximum close to $\omega_{p}$. Beyond the percolation threshold, the spectrum acquires a second, low-frequency peak (the presence of two plasmons modes in metallic shells was first predicted long $\mathrm{ago}^{21}$ ) whose frequency increases as $C$ increases further. In this connection it is worth noting that splitting of a single plasmon peak is a quite general phenomenon observed at any deviation from spherical symmetry, e.g., in ellipsoidal particles, ${ }^{22}$ periodic arrays, ${ }^{23}$ or clusters $^{24}$ of spherical particles.

If we assume that our model describes evolution of nanodots in the process of metallic shell growth, then, after the dot surface is covered completely, that is $C \rightarrow 1$, further growth will increase $r_{2}$, that is $P$. This is illustrated by two last curves in Fig. 3 obtained for $C=1$ and for increasing values of $P$. As it is evident, the two peaks approach each other and merge eventually in the vicinity of $\omega_{p} / \sqrt{3}$. The total evolution of absorption spectra shown in Fig. 3 is in good qualitative agreement with the experimental observations for nanoshells with $\mathrm{SiO}_{2}$ and $\mathrm{Ag}_{2} \mathrm{~S}$ cores. ${ }^{12,13}$ They include initial red followed by a blueshift of the peak and transformation from a single- to a double-peak absorption spectra with different amplitude of peaks in the course of gold coating the nanoparticle surface.

It is interesting that similar calculations for $\varepsilon_{1} \simeq 10$ typical for semiconductor cores, show no qualitative distinctions with the results for $\varepsilon_{1}=3$. On the contrary, the curves with $\varepsilon_{1}=1$ (hollow nanoshells), drawn by dashed lines in Fig. 3, demonstrate an opposite character of asymmetry at intermediate $C$.

Due to a strong frequency dispersion of the shell dielectric constant, as well as of the effective $\varepsilon$ of the whole system, the spatial distribution of ac electric field caused by external illumination will depend noticeably on the light frequency $\omega$, if the latter falls within the spectral region covering the effective plasmon frequencies. In particular, the electric field in the core $\mathbf{E}_{1}$ (which is uniform throughout the whole core) is given by

$$
\mathbf{E}_{1}=\frac{9 \varepsilon_{\mathrm{eff}}^{(2)}}{2\left[\varepsilon_{\mathrm{eff}}^{(2)}\right]^{2} P+\varepsilon_{\mathrm{eff}}^{(2)}\left(3 \varepsilon_{1}-2 \varepsilon_{1} P+6-2 P\right)+2 \varepsilon_{1} P} \mathbf{E}_{0},
$$

where $\mathbf{E}_{0}$ is the field strength far from the nanostructure (see Ref. 18).

Figure 4 shows the "amplification factor" of electric field $A(\omega) \equiv\left|E_{1} / E_{10}\right|$, where $E_{10}=3 E_{0} /\left(2+\varepsilon_{1}\right)$ is the field which would exist in a core containing no shell. Below the percolation threshold (at $C<C_{p}$ ) this factor differs from 1 by less than $20 \%$, which has no practical interest, and this region is not shown in the figure. At larger $C, A(\omega)$ acquires two sharp peaks: at $\omega$ slightly less than $\omega_{p}$ and at essentially lower $\omega$. Comparison with Fig. 3 shows that they correspond to the frequencies of two plasmons existing in the system.

The maximum field amplification in the peaks depends drastically on the electron scattering in shells described by the parameter $\nu$. It is distinctly seen from comparison of solid and dashed curves in Fig. 4 calculated for two different values of $\nu$. For this reason, it is important to know $\nu$ in real experimental samples. The widely used approach based on estimating $\nu$ from the mobility data in bulk metal seems not enough adequate due to a possible distinction of crystal quality in bulk and nanostructures. However, the detailed experimental work ${ }^{25}$ contains data on the electron mean free path in $\mathrm{Ag}$ nanoshell, which varies from 1 to $2 \mathrm{~nm}$. This corresponds to $\nu / \omega_{p} \simeq 0.05-0.1$, which, according to Fig. 4, is enough for observation of noticeable amplification for nanoshells with a sufficiently large $C$. Note that at $C<C_{p}$ the core electric field $E_{1}$ is much less sensitive to $\nu$ and cannot be noticeably increased even by a large decrease in $\nu$.

Our description is based on the Drude approximation containing two parameters: $\omega_{p}$ and $\nu$. Such an approach, fully applicable for simple, free-electron-like metals, such as Na, is also often used for metals with a complex Fermi surface by including in $\varepsilon_{M}$ some constant term describing the polarizability of $d$ electrons (see, e.g., Ref. 24), which results in renormalization of $\omega_{p}$ making it 2-3 times lower than the free-electron value. As to $\nu$ in small nanocrystals, it may contain an additional term inversely proportional to their radius $^{22,24}$ and, in addition, implicitly depend on $C$ since the merging of small islands may decrease the role of surface scattering. That is why $\omega_{p}$ and $\nu$ may not quantitatively coincide with those for free electrons in bulk materials but rather should be considered as phenomenological parameters to be found from the best agreement with experiments.

A direct method of observing the effects discussed in this section consists in measuring the luminescence excitation spectra of semiconductor quantum dots surrounded by metallic nanoshells. If the effective band gap of quantum dots is less than the characteristic plasmon energy, light causing plasmon absorption provides excitation of luminescence in dots. The intensity of luminescence will be proportional to the intensity of excitation, which, in turn, is proportional to the square of ac electric field in a dot (if the density of states in a dot does nor vary dramatically in the considered interval of excitation frequencies). According to Fig. 4, this electric field strongly depends on the light frequency and, as a result, the excitation spectra should have two sharp maxima (or a single maximum if the luminescent line lies between two plasmon frequencies).

\section{NANOSHELLS WITH LUMINESCENT CORE}

We have already mentioned a possible role of nanoshells in creating effectively luminescent quantum dots by amplifying ac electric field of the exciting light due to the influence of image forces. Now we discuss the influence of nanoshells on the light emitted by a semiconductor core. The situation is similar to that considered in Sec. III. The ac electric field emitted by a core, which can be considered as an effective dipole $\mathbf{d}_{0}$, is disturbed by a metallic shell so that the field far from the nanostructure looks similar to the one created by some other effective dipole d. By analogy with Sec. III, we may expect that in the vicinity of a plasmon resonance the condition $\left|d / d_{0}\right| \gg 1$ can be realized, which means an effective amplification of the emitted radiation as well. 

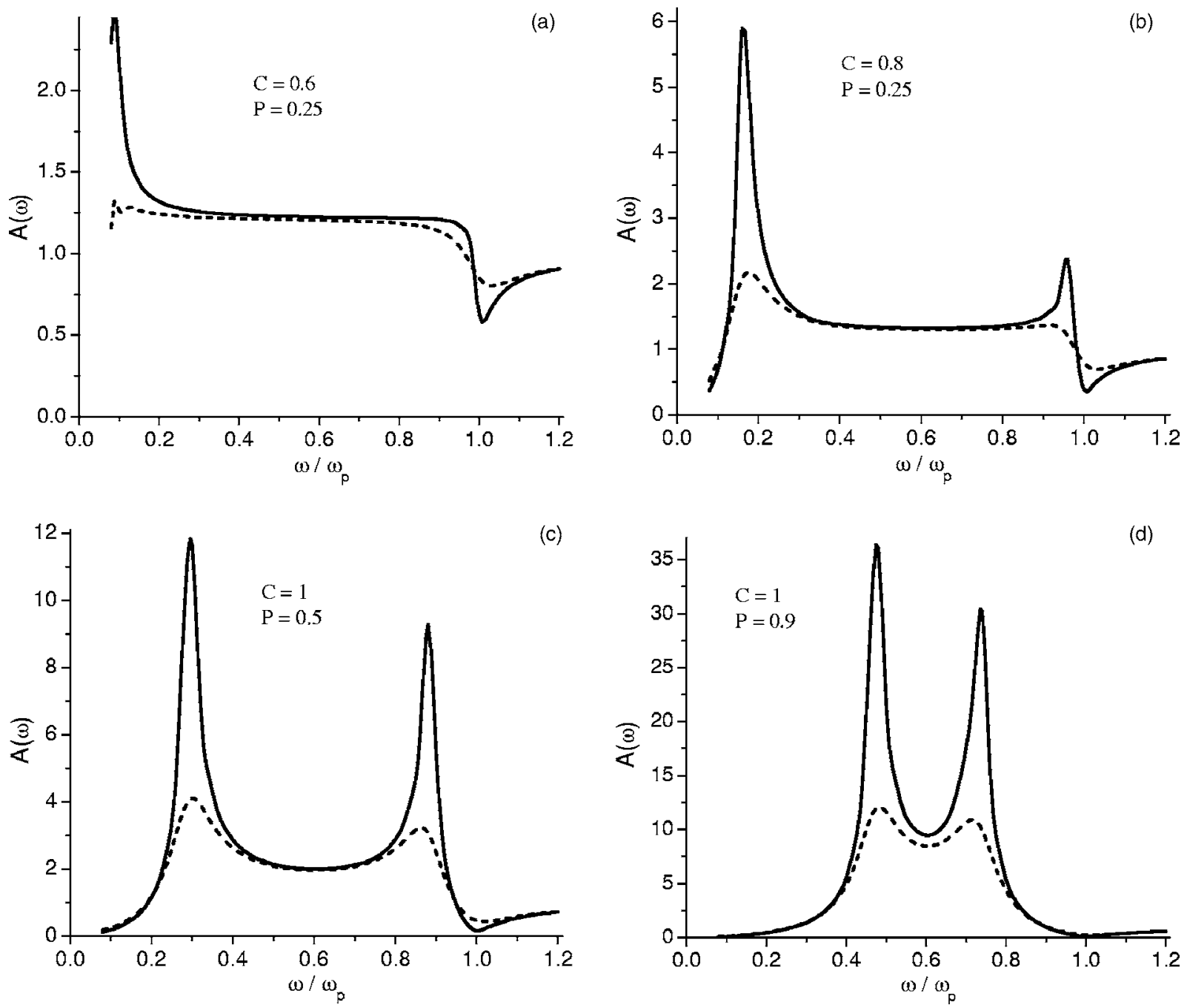

FIG. 4. Spectra of electric field amplification in metallic nanoshells with $C=0.6, P=0.25$ (a); $C=0.8, P=0.25$ (b); $C=1, P=0.5$ (c); $C$ $=1, P=0.9$ (d), for $\nu / \omega_{p}=0.03$ (solid) and $\nu / \omega_{p}=0.1$ (dashed). Figures (a)-(d) correspond, respectively, to the absorption spectra of Figs. 3(c)-3(f).

Generally speaking, the problem of an emitting dipole inside a spherical shell is rather cumbersome. ${ }^{26,27}$ However, our case is characterized by complete spherical symmetry so that we may consider $\mathbf{d}_{0}$ to be placed in the sphere center, after which the problem is simplified dramatically and becomes similar to that used in Ref. 18 for calculation of $\mathbf{E}_{1}$ created in a core by an external field $\mathbf{E}_{0}$ and resulted in Eq. (4). In our case the situation is in some sense reciprocal: we have an active dipole $\mathbf{d}_{0}$ inside the core but no uniform field at large distance. A general solution of the Laplace equation with the same angular dependence as the dipole's potential has the form $\varphi(\mathbf{r})=\left(a r+b / r^{2}\right) \cos \theta$. At $r<r_{1}, b=d_{0}$ while $a$ is some unknown field $E_{1}$. At $r_{1}<r<r_{2}$ both coefficients are unknown, and at $r>r_{2}, a=0$ while the role of $b$ is played by the effective dipole $d$ we want to determine. The continuity of potential $\varphi$ and electrical induction $\varepsilon(\partial \varphi / \partial r)$ at $r=r_{1}$ and $r=r_{2}$ gives us the necessary system of equations for finding all coefficient and, particularly, the "amplification factor" $\left|d / d_{0}\right|$. Calculations show that it has the same value

$$
A(\omega)=\left|\frac{9 \varepsilon_{\mathrm{eff}}^{(2)}}{2\left[\varepsilon_{\mathrm{eff}}^{(2)}\right]^{2} P+\varepsilon_{\mathrm{eff}}^{(2)}\left(3 \varepsilon_{1}-2 \varepsilon_{1} P+6-2 P\right)+2 \varepsilon_{1} P}\right|
$$

as for the electric field [see Eq. (4)] and hence Fig. 4 shows the amplification spectra of not only the excitation but of the luminescence signal as well.

The real luminescence spectra of quantum dots with metallic shells, given by the spectral dependences of $|d(\omega)|^{2}$, are determined by the bare luminescence spectrum of dots $\left|d_{0}(\omega)\right|^{2}$ and by the spectrum of $A^{2}(\omega)$. To demonstrate the role of metallic shell, we performed calculations assuming $\left|d_{0}(\omega)\right|^{2}$ to have a Gaussian shape with the center at $\omega_{\mathrm{em}}$ $=0.3 \omega_{p}$ and the width $0.1 \omega_{p}$. One can see from Fig. 4 that for reasonable parameters of nanoshells the lower plasmon peak may lie both left and right of this $\omega_{\mathrm{em}}$. Figure 5 shows the resulting transformation of the luminescence spectrum. The most remarkable but quite expectable result consists in a dramatic increase in the luminescence intensity near the reso- 


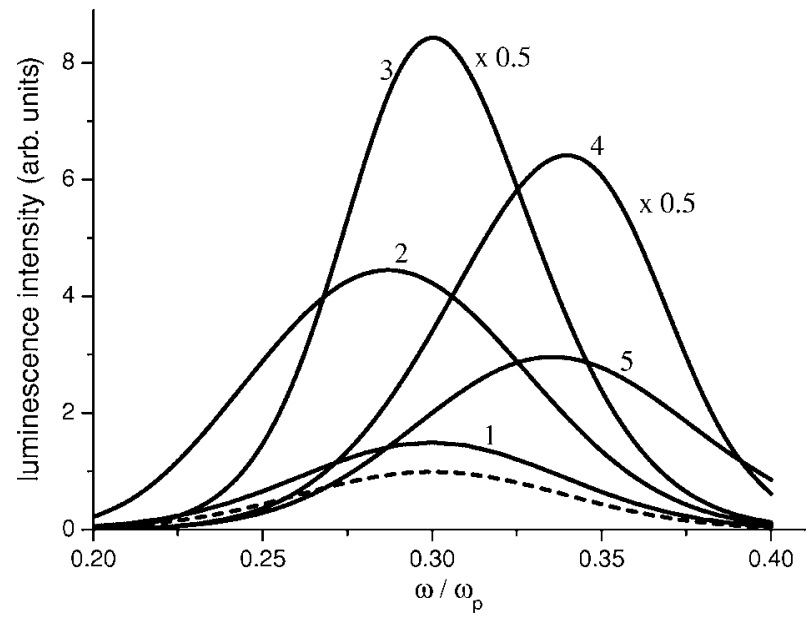

FIG. 5. Amplification and spectral shift of a luminescence line with $\omega_{\mathrm{em}}=0.3 \omega_{p}$ in a core-shell structure with $C=0.6, P=0.25$, curve 1 [see also Figs. 3(c) and 4(a)]; $C=1, P=0.25$, curve 2; $C$ $=1, P=0.5$, curve 3 [see also Figs. $3(\mathrm{e})$ and $4(\mathrm{c})$ ]; $C=1, P=0.7$, curve 4; $C=1, P=0.9$, curve 5 [see also Figs. 3(f) and 4(d)], for $\nu / \omega_{p}=0.1$. The dashed curve shows the bare luminescent line.

nant condition when the plasmon frequency coincides with $\omega_{\mathrm{em}}$. Another interesting feature consists in shifting the frequency of luminescence line in off-resonant conditions. Convolution of a bare luminescence line with the amplification spectrum of Fig. 4, whose position, amplitude and width depend on $C$ and $P$, may result in a nonmonotonic behavior of the resulting spectrum, as shown in Fig. 5. From the practical point of view it means that for the same quantum dot the increase of a nanoshell thickness (which grows with the curve number) may cause first red (curves 1,2), then blue (curves 2-4), and then again slight redshift (curves 4,5) of the luminescence line.
It would be very attrative to enhance luminescence dramatically by a simultaneous amplification of both exciting and emitting light. Such situation often occurs in SERS but in luminescence its realization is more difficult since the difference in frequencies of exciting $\omega_{\mathrm{ex}}$ and emitted $\omega_{\mathrm{em}}$ light is usually quite large. They can be covered by a single plasmon peak only if the latter is wide enough, which, according to Fig. 4, corresponds to a low level of amplification. An alternative possibility consists in using both plasmon peaks shown in Fig. 4. In this case the shell parameters should be chosen in such a way that the low-frequency peak corresponds to $\omega_{\mathrm{em}}$ in a given quantum dot while the source of excitation is tuned for $\omega_{\text {ex }}$ to coincide with the second peak lying near $\omega_{p}$, which for most metals belongs to the untraviolet spectral region.

\section{CONCLUSION}

We have analyzed the optical properties of dielectric nanoparticles covered by metallic shells, which can be either continuous or consist of separate nonconnected clusters, such as, e.g., at the initial stage of growth. Evolution of these properties was studied as a function of the metal concentration within the framework of the two-dimensional EMA. The results are applied to the problem of enhancing the luminescence characteristics of semiconductor nanocrystals covered by metallic nanoshells.

In conclusion, we also mention that the formulas obtained remain adequate to moderate nanodot concentrations. The formula for polarizability (3) assumes induced dipole moments neglecting higher multipoles, which is possible if the distance between nanodots essentially exceeds their radius. If this assumption fails (at high nanodot density $N$ ), we can again apply the EMA, this time for a statistical mixture of nanodots with polarizability Eq. (3) and dielectric $\varepsilon_{D}$, using the three-dimensional EMA.
${ }^{1}$ Z. Liu, H. Wang, H. Li, and X. Wang, Appl. Phys. Lett. 72, 1823 (1998).

${ }^{2}$ S. Cho et al., Thin Solid Films 377-378, 97 (2000).

${ }^{3}$ S. K. Mandal, R. K. Roy, and A. K. Pal, J. Phys. D 35, 2198 (2002).

${ }^{4}$ P. Hanarp, M. Käll, and D. S. Sutherland, J. Phys. Chem. B 107, 5768 (2003).

${ }^{5}$ R. K. Roy, S. Bandyopadhyaya, and A. K. Pal, Eur. Phys. J. B 39, 491 (2004).

${ }^{6}$ K.-H. Su, Q.-H. Wei, X. Zhang, J. J. Mock, D. R. Smith, and S. Schultz, Nano Lett. 3, 1087 (2003).

${ }^{7}$ I. Webman, J. Jortner, and M. H. Cohen, Phys. Rev. B 15, 5712 (1977).

${ }^{8}$ C. J. Adkins, J. Phys. C 12, 3389 (1979).

${ }^{9}$ B. I. Shklovskii and A. L. Efros, Electronic Properties of Doped Semiconductors (Springer, Berlin, 1986).

${ }^{10}$ M. R. Melloch, J. M. Woodall, E. S. Harmon, N. Otsuka, F. H. Pollak, D. D. Nolte, R. M. Feenstra, and M. A. Lutz, Annu. Rev. Mater. Sci. 25, 547 (1995).
${ }^{11}$ H. Ruda and A. Shik, Phys. Rev. B 63, 085203 (2001).

${ }^{12}$ S. J. Oldenburg, R. D. Averitt, S. L. Westcott, and N. J. Halas, Chem. Phys. Lett. 288, 243 (1998).

${ }^{13}$ H. S. Zhou, I. Honma, H. Komiyama, and J. W. Haus, Phys. Rev. B 50, 12052 (1994).

${ }^{14}$ Y. Sun, B. Mayers, and Y. Xia, Adv. Mater. (Weinheim, Ger.) 15, 641 (2003).

${ }^{15}$ M. Moskovits, Rev. Mod. Phys. 57, 783 (1985).

${ }^{16}$ L. Bakueva, S. Musikhin, E. H. Sargent, H. E. Ruda, and A. Shik, in Handbook of Organic-Inorganic Hybrid Materials and Nanocomposites, edited by H. S. Nalwa (American Scientific, New York, 2003).

${ }^{17}$ X. Michalet, F. Pinaud, T. D. Lacoste, M. Dahan, M. P. Bruchez, A. P. Alivisatos, and S. Weiss, Single Mol. 2, 261 (2001).

${ }^{18}$ A. E. Neeves and M. H. Birnboim, J. Opt. Soc. Am. B 6, 787 (1989).

${ }^{19}$ E. Prodan, P. Nordlander, and N. J. Halas, Chem. Phys. Lett. 368, 94 (2003).

${ }^{20}$ V. S. Zuev, A. V. Frantsesson, D. V. Vlasov, and G. Ya. Zueva, 
Opt. Spectrosc. 96, 426 (2004).

${ }^{21}$ G. Mukhopadhyay and S. Lundqvist, Nuovo Cimento Soc. Ital. Fis., B 27, 1 (1975).

${ }^{22}$ U. Kreibig and M. Vollmer, Optical Properties of Metal Clusters (Springer, Berlin, 1995).

${ }^{23}$ A. Taleb, V. Russier, A. Courty, and M. P. Pileni, Phys. Rev. B 59, 13350 (1999).
${ }^{24}$ V. Russier and M. P. Pileni, Surf. Sci. 425, 313 (1999).

${ }^{25}$ P. Mulvaney, Langmuir 12, 788 (1996).

${ }^{26}$ H. Chew, P. J. McNutty, and M. Kerker, Phys. Rev. A 13, 396 (1976).

${ }^{27}$ H. Chew, M. Kerker, and P. J. McNutty, J. Opt. Soc. Am. 66, 440 (1976). 